\title{
Transcranial direct current stimulation enhances theory of mind in Parkinson's disease patients with mild cognitive impairment: a randomized, double-blind, sham-controlled study
}

\author{
Mauro Adenzato ${ }^{1,2+}$, Rosa Manenti ${ }^{3 \dagger}$, Ivan Enrici ${ }^{4^{*}}$ (D) Elena Gobbi ${ }^{3}$, Michela Brambilla ${ }^{3}$, Antonella Alberici ${ }^{5}$, \\ Maria Sofia Cotelli ${ }^{5}$, Alessandro Padovani ${ }^{5}$, Barbara Borroni ${ }^{5}$ and Maria Cotelli ${ }^{3}$
}

\begin{abstract}
Background: Parkinson's Disease (PD) with mild cognitive impairment (MCI) (PD-MCl) represents one of the most dreaded complications for patients with PD and is associated with a higher risk of developing dementia. Although transcranial direct current stimulation (tDCS) has been demonstrated to improve motor and non-motor symptoms in PD, to date, no study has investigated the effects of tDCS on Theory of Mind (ToM), i.e., the ability to understand and predict other people's behaviours, in PD-MCl.

Methods: In this randomized, double-blind, sham-controlled study, we applied active tDCS over the medial frontal cortex (MFC) to modulate ToM performance in twenty patients with PD-MCl. Twenty matched healthy controls (HC) were also enrolled and were asked to perform the ToM task without receiving tDCS.

Results: In the patients with PD-MCl, i) ToM performance was worse than that in the HC, ii) ToM abilities were poorer in those with fronto-executive difficulties, and iii) tDCS over the MFC led to significant shortening of latency for ToM tasks.

Conclusions: We show for the first time that active tDCS over the MFC enhances ToM in patients with PD-MCl, and suggest that non-invasive brain stimulation could be used to ameliorate ToM deficits observed in these patients.
\end{abstract}

Keywords: Medial frontal cortex (MFC), Mild cognitive impairment (MCl), Parkinson's disease (PD), Theory of mind (ToM), Transcranial direct current stimulation (tDCS)

\section{Background}

Parkinson's Disease (PD) is the second most common neurodegenerative disease [1]. Its cardinal clinical manifestations are motors dysfunctions, including resting tremor, muscle rigidity, akinesia, and postural instability [2-6]. However, PD is also associated with non-motor deficits, including cognitive and emotional impairments, autonomic dysfunction, sleep disorders, neuropsychiatric

\footnotetext{
* Correspondence: ivan.enrici@unito.it

${ }^{\dagger}$ Mauro Adenzato and Rosa Manenti contributed equally to this work.

${ }^{4}$ Department of Philosophy and Educational Sciences, University of Turin, via Gaudenzio Ferrari 9, 10124 Turin, Italy

Full list of author information is available at the end of the article
}

disorders, and sensory impairment [3, 4, 7-16]. Recent studies have suggested that these symptoms are the major determinants of quality of life in patients with PD [13, 17].

In recent years, there has been great interest in the early phases of $\mathrm{PD}$. In particular, PD-mild cognitive impairment (PD-MCI) represents a transitional state between normal aging and dementia in patients with PD. This condition is associated with a higher risk of developing PD with dementia and represents one of the most dreaded complications of the disease for patients and caregivers [18]. Interestingly, PD-MCI prevalence seems to range from 25 to $30 \%$ among non-demented patients with PD [19]. PD-MCI is a heterogeneous clinical

(c) The Author(s). 2019 Open Access This article is distributed under the terms of the Creative Commons Attribution 4.0 International License (http://creativecommons.org/licenses/by/4.0/), which permits unrestricted use, distribution, and 
condition and is generally characterized by impairments in cognitive domains such as memory, visuospatial function, and frontal/executive functions, with preserved activities of daily living [3, 4, 10, 19-23].

Research on the social cognitive profile of PD has revealed disturbances in Theory of Mind (ToM) ability, i.e., the ability to attribute mental states to others and to predict, describe, and explain behaviour on the basis of such mental states [24-29]. Imaging studies have suggested that ToM ability relies on a distributed neural network including the right and left temporo-parietal junctions, the precuneus, and the medial frontal cortex (MFC) [30, 31]. Several studies have suggested a pivotal role for the MFC in ToM (for a review, see [32]).

ToM has attracted considerable interest in neurodegenerative diseases in recent years [33-39]. In particular, ToM difficulties recorded in patients with PD in the early stages of the disease principally involve the cognitive component of ToM (i.e., inferences about others' beliefs and intentions). The spatio-temporal progression of dopamine depletion in PD supports the hypothesis that the affective component (i.e., inferences about others' emotions and feelings) may only be impaired in the advanced stages of the disease when depletion of dopamine in the striatum and the consequent hypostimulation of the MFC also involve more medial portions of this brain region (the dorsolateral portion of MFC is affected in early PD stages). Interestingly, it has been proposed that difficulties in the cognitive component of ToM, which are detected in ToM tasks in patients with $\mathrm{PD}$, could be partially explained by the executive function impairment characterizing the disease [38].

In this study, we applied anodal transcranial direct current stimulation (tDCS) over the MFC (Fpz site, with the cathode between $\mathrm{Oz}$ and Inion) to modulate $\mathrm{ToM}$ performance in patients with PD-MCI. tDCS is a non-invasive stimulation technique wherein weak polarizing electrical current externally applied through a pair of electrodes attached to the head for a few minutes can generate a change in neuronal excitability and consequently modulate cognitive task performance. Depending on the polarity of the current flow, brain excitability can be increased (anode) or decreased (cathode) [40-42].

Several studies have reported short-term modulation of cognitive performance in typical populations and in patients using tDCS [43-45]. In addition, a single tDCS session has been demonstrated to have an effect on social cognitive abilities in healthy subjects [46-53]. Although anodal tDCS has already been demonstrated to improve motor and non-motor symptoms in PD [54-60], to the best of our knowledge, no study has yet investigated the effects of tDCS on ToM abilities in patients with PD-MCI.

To evaluate the impact of active tDCS on ToM performance in patients with $\mathrm{PD}-\mathrm{MCI}$, we used a video-tape version of a cognitive ToM task to investigate the ability to represent other individuals' private and communicative intentions based on the observation of daily actions [61-64]. We investigated whether the application of active tDCS over the MFC (Fpz site, with the cathode between $\mathrm{Oz}$ and Inion) would facilitate ToM performance in this study with a double-blind, sham-controlled experimental design. Based on the PD cognitive profile described, we hypothesized that, in patients with PD-MCI, ToM task performance would i) be worse than it is in healthy controls, ii) correlate with executive functioning, and iii) be enhanced by active tDCS over the MFC when compared to sham.

\section{Materials and methods}

Participants and control group

Twenty patients fulfilling the UK Parkinson's Disease Society Brain Bank clinical diagnostic criteria for PD $[65,66]$ were consecutively recruited at IRCCS Istituto Centro San Giovanni di Dio Fatebenefratelli in Brescia, Italy. Patients with PD-MCI were classified using the Parkinson's Disease Cognitive Rating Scale (PD-CRS) (PD-MCI score range $=65-81$ [67]) and using comprehensive neuropsychological testing to assess different cognitive domains (see below) [3].

The exclusion criteria included a) other neurological and psychiatric disorders; b) history of traumatic brain injury; c) clinically known hearing or vision impairment or a past history of alcohol abuse; d) diagnosis of PD-dementia [67] or Mini-Mental Parkinson (MMP) score < $25[68,69]$; and e) any contraindication for tDCS [70]. All patients had been on stable pharmacological therapy for at least 6 months prior to entering the study.

Twenty matched healthy controls ( $\mathrm{HC}$ ) were also enrolled in the study for behavioural assessment of their ToM abilities ( 11 women and 9 men, mean age $=69.4$ \pm 5.8 years, mean education $=11.6 \pm 4.4$ years). The inclusion criteria for the $\mathrm{HC}$ were as follows: no history of mental illness or cognitive decline, no motor or cognitive complaints, normal objective cognitive performance in all of the administered neuropsychological tests, normal scores in functional assessment, and absence of mood and anxiety disorders. The $\mathrm{HC}$ were asked to perform the ToM task without tDCS in order to allow us to compare ToM abilities in patients with PD-MCI to those in HC.

All participants were made fully aware of the aims of the research, and written informed consent was obtained from all subjects. The study was approved by the local ethics committee (IRCCS Istituto Centro San Giovanni di Dio Fatebenefratelli, Brescia, Italy) and was conducted in accordance with the Declaration of Helsinki. 


\section{Procedure}

\section{Clinical and neuropsychological assessment}

All patients underwent extensive clinical and neuropsychological evaluations carried out over two sessions.

A comprehensive neuropsychological assessment based on the previously published recommendations is used $[3,69]$. The cognitive tests battery included test for assessing global cognitive abilities (PD-CRS [71-73]) and tests for memory, language, and attentional and executive functions. We used MMP as a brief screening test for global cognition $[66,68,74,75]$. In addition, PD-CRS is applicable to all stages of PD both for routine clinical practice and for data collection in clinical trials and it is rated as "recommended" by the International Parkinson and Movement Disorder Society [76-78].

The neuropsychological test battery included measures used to assess verbal fluency (phonemic and semantic), object and action naming (International Picture Naming Project, [79]), attention and executive functions (Trail Making Test, Test of Attentional Performance [TEA] [80], Stroop Test, Frontal Assessment Battery [FAB] [81]), and memory (Rey Auditory Verbal Learning Test, immediate and delayed recall, and Digit Span Forward and Backward). All of the tests were administered and scored according to standard procedures [82]. Results of the neuropsychological assessment of the patients with PD-MCI are presented in Table 1.

Depressive symptoms were assessed using the Beck Depression Inventory-II (BDI-II; [83]). Clinical evaluation included the Parkinson's Disease Quality of Life Questionnaire-39 [84], the Barratt Impulsivity Scale [85], the Apathy Evaluation Scale [86], and the Rapid Eye Movement Sleep Behavior Disorders Screening Questionnaire (RBDSQ; [83-85, 87]). The Unified Parkinson's Disease Rating Scale (UPDRS-III; [88]), the Italian version of the 20-Item Toronto Alexithymia Scale (TAS 20; [89]), the Interpersonal Reactivity Index [90], and the Hoehn \& Yahr Scale [91] were also administered. The demographic and clinical characteristics of the patients with PD-MCI are presented in Table 2.

The present work was a randomized, double-blind, sham-controlled study. Each patient received both active and sham tDCS over the MFC (Fpz site, with the cathode between $\mathrm{Oz}$ and Inion) in two different sessions separated by at least 2 days and in randomized order. All patients and the experimenter were blind to the type of tDCS applied. During each session, a video version of a cognitive ToM task was administered. According to the literature, tDCS excitability changes induced by one session of tDCS applied for a few minutes are expected to last for up to $1 \mathrm{~h}$ [92-94]. Therefore, the performance of the patients was expected to return to its initial level between the two sessions of stimulation.

\section{Theory of mind tasks}

All of the participants performed two ToM tasks: the Reading the Mind in the Eyes (RME) task, which was used to assess individual ToM abilities, and the Attribution of Intentions (AI) task, which was used to test the effects of tDCS on cognitive ToM ability.

The RME is an advanced ToM task evaluating the subject's ability to represent others' mental states by observing only their eyes [95]. The participants were required to choose which word, among four options, better described what the character in the photograph was thinking or feeling. The total number of correct choices represented the RME score. Participants were administered the RME before the tDCS brain stimulation.

The AI task is a video version of a cognitive ToM task previously used in young healthy individuals [46]. Participants were asked to demonstrate their comprehension of the displayed stories (short videos lasting 1500 milliseconds) by choosing the appropriate story ending (out of two concluding pictures displayed until the response). The two possible story endings were shown simultaneously until the participant responded by pressing the corresponding button on the button box as quickly as possible. The correct picture represented a probable conclusion, whereas the incorrect picture represented an improbable ending (see Fig. 1).

The two experimental conditions were a) the Private Intention condition (PInt), in which participants were required to recognize another person's intention while watching his/her isolated actions, e.g., hanging a picture on the wall; and b) the Communicative Intention condition (CInt), in which participants were required to recognize another person's communicative intention during a social interaction, e.g., asking another person to obtain a glass of water for them.

We displayed 34 video stories for each condition, for a total of 68 stories using Presentation software (Version 16.3, www.neurobs.com) running on a personal computer with a 15-in. screen. Participants were seated in a quiet room in front of a computer monitor placed $60 \mathrm{~cm}$ away from them. The visual location (right and left side of the screen) of the correct answer was randomized. Accuracy was recorded as the number of correct trials. The reaction time (RT) for each correct response was recorded in milliseconds from the onset of the presentation of the two concluding pictures until detection of the response. The items were divided into two blocks (17 PInt and 17 CInt stimuli each) corresponding to the two types of stimulation (active and sham stimulation). Two additional stimuli for each condition were selected for use in a training session. The stimulation conditions and the order of the presentation of the two blocks were randomized across participants. The tests were 
Table 1 Neuropsychological assessment of patients with Parkinson's Disease-mild cognitive impairment $(n=20)$

\begin{tabular}{|c|c|c|}
\hline & Mean (SD) & Cut-off \\
\hline \multicolumn{3}{|l|}{ Screening for dementia } \\
\hline Mini Mental Parkinson & $28.0(2.7)$ & $\geq 22.85$ \\
\hline \multicolumn{3}{|l|}{ Global cognitive abilities } \\
\hline \multicolumn{3}{|l|}{ Parkinson's Disease-Cognitive Rating Scale (PD-CRS) } \\
\hline PD-CRS Total Score (max: 134) & $78.9(15.6)$ & $\geq 82$ \\
\hline PD-CRS Cortical Score (max: 30) & $26.1(2.4)$ & - \\
\hline PD-CRS Frontal Subcortical Score (max: 104) & $52.8(14.0)$ & - \\
\hline \multicolumn{3}{|l|}{ Memory } \\
\hline Rey Auditory Verbal Learning Test, immediate recall & $35.8(11.1)$ & $>28.52$ \\
\hline Rey Auditory Verbal Learning Test, delayed recall & $7.0(3.5)$ & $>4.68$ \\
\hline Digit Span (Forward) & $5.5(0.9)$ & $>4.25$ \\
\hline Digit Span (Backward) & $4.1(0.9)$ & $>2.64$ \\
\hline \multicolumn{3}{|l|}{ Language } \\
\hline Verbal Fluency, phonemic & $30.6(9.9)$ & $>16$ \\
\hline Verbal Fluency, semantic & $37.2(9.0)$ & $>24$ \\
\hline Objects Picture Naming task (International Picture Naming Project (IPNP), \%) & $69.1(11.9)$ & - \\
\hline Actions Picture Naming task (IPNP, \%) & $87.3(14.2)$ & - \\
\hline \multicolumn{3}{|l|}{ Attentional and Executive Functions } \\
\hline Frontal Assessment Battery & $15.3(2.5)$ & $>13.4$ \\
\hline Stroop test (interference effect on time, seconds) & $32.4(14.1)$ & $<36.92$ \\
\hline Stroop test (interference effect on error number) & $2.4(3.3)$ & $<4.24$ \\
\hline Trail Making Test, part A (seconds) & $51.8(26.5)$ & $<94$ \\
\hline Trail Making Test, part B (seconds) & $184.7(125.7)$ & $<283$ \\
\hline \multicolumn{3}{|l|}{ Test of Attentional Performance } \\
\hline Go/NoGo (time, ms) & $528.9(88.7)$ & - \\
\hline Go/NoGo (accuracy) & $28.2(2.5)$ & - \\
\hline Working Memory (time, milliseconds) & $803.6(192.5)$ & - \\
\hline Working Memory (accuracy) & $11.7(1.1)$ & - \\
\hline Response Flexibility (time, milliseconds) & $1390.4(512.2)$ & - \\
\hline Response Flexibility (accuracy) & $78.0(17.8)$ & - \\
\hline
\end{tabular}

Raw scores are reported. Standard deviation (SD) is presented in parentheses

Cut-off scores according to Italian normative data are reported

Bold data indicate scores below the cut-off value

administered on two consecutive days at the same time of the day to minimize the likelihood of interference from confounding factors.

\section{tDCS procedure}

Active tDCS was applied using a battery-driven constant-current stimulator (BrainStim, EMS; Bologna, Italy) through a pair of saline-soaked sponge electrodes $(7 \mathrm{~cm} \times 5 \mathrm{~cm})$. The target area for tDCS was the MFC (Montreal National Institute coordinates: 0, 60, 18), as it has been recognized as a pivotal region in intention processing [62-64, 96, 97]. In order to apply tDCS to the MFC, we placed the anode over Fpz and the cathode between Inion and $\mathrm{Oz}$ (Fig. 1) according to the 10-20 electroencephalography international system [98]. During active tDCS, a constant current of $1.5 \mathrm{~mA}$ was applied for $6 \mathrm{~min}$ (with a ramping period of $10 \mathrm{~s}$ at the beginning of the stimulation), starting $2 \mathrm{~min}$ before the beginning of the AI task and covering the entire duration of the task. The current density $\left(0.043 \mathrm{~mA} / \mathrm{cm}^{2}\right)$ was maintained below the safety limits [70]. In the sham stimulation condition, the tDCS procedure was the same, but the current was turned off $10 \mathrm{~s}$ after the beginning of the stimulation (not including the durations of the fade-in and fade-out periods $=10 \mathrm{~s}$ ) and turned on for the last $10 \mathrm{~s}$ of the stimulation period. Therefore, the 
Table 2 Demographic and clinical characteristics of patients with Parkinson's Disease-mild cognitive impairment $(n=20)$

\begin{tabular}{ll}
\hline & Mean (SD) \\
\hline Age (years) & $65.6(8.4)$ \\
Education (years) & $10.3(4.6)$ \\
Sex (male/female) & $10 / 10$ \\
Age of onset (years) & $58.9(7.5)$ \\
Levodopa-equivalent daily dose (mg) & $555.7(323.1)$ \\
Cumulative IIIness Rating Scale - severity & $1.6(0.3)$ \\
Cumulative IIIness Rating Scale - comorbidity & $2.9(1.8)$ \\
Unified Parkinson's Disease Rating Scale III & $24.1(9.5)$ \\
Hoehn \& Yahr & $1.8(0.7)$ \\
Toronto Alexithymia Scale-20 & $50.7(14.8)$ \\
Interpersonal Reactivity Index & $84.4(13.0)$ \\
REM Sleep Behavior Disorder Screening Questionnaire & $3.6(2.4)$ \\
Parkinson's Disease Quality of Life Questionnaire & $23.2(13.0)$ \\
Beck Depression Inventory II & $11.7(6.0)$ \\
Apathy Evaluation Scale & $12.1(10.2)$ \\
Barratt Impulsivity Scale-11 & $60.5(8.1)$ \\
\hline Standard devaton (SD) is pren
\end{tabular}

Standard deviation (SD) is presented in parentheses

participants experienced an itching sensation below the electrodes at the beginning and end of the stimulation, making this condition indistinguishable from the experimental stimulation.

The study was a randomized double-blind experiment: the participants and the experimenter were not aware of the stimulation that was delivered. The two AI stimuli sessions corresponded to the two tDCS conditions: active and sham tDCS. The stimulation conditions were randomized across participants and executed on two different days at the same time of day to minimize the likelihood of interference from confounding factors. Half of the male and female participants received active stimulation on day 1 and sham stimulation on day 2, while the other half of the participants received sham stimulation on day 1 and active stimulation day 2. Active or sham tDCS were delivered after a numeric code was input into the device. This step allowed for blinding of the operator before and during the tDCS administration.

In order to detect differences in the perception of sensations, to blind the participants to the type of stimulation they were receiving, and to register potential side effects of tDCS, at the end of the stimulation session we asked the participants to answer a questionnaire regarding the perceptual sensations they experienced during the active and sham tDCS experiments.

\section{Statistical analyses}

Statistical analyses were performed using Statistica software (version 10; www.statsoft.com/Products/
STATISTICA-Features). Since the data were normally distributed (RTs, Kolmogorov-Smirnov Test: $\mathrm{d}=0.19, p$ $=0.21$; and accuracy, Kolmogorov-Smirnov Test: $\mathrm{d}=$ $0.16, p>0.25$ ), AI task performance (accuracy and RT) was analysed using parametric analyses.

First, AI task performance (accuracy and RT) in the PD-MCI group under the sham tDCS condition was compared to that in an age-, sex-, and education-matched $\mathrm{HC}$ group using analysis of variance (ANOVA) with the type of stimulus (PInt or CInt) as the within-participant factors and group (PD or $\mathrm{HC}$ ) as the between-participants factors. We compared the RME scores of the PD-MCI and $\mathrm{HC}$ groups using a one-way ANOVA.

Subsequently, PD-MCI AI task performance (accuracy and RT) was analysed using repeated-measures ANOVA, with the type of stimulation (active or sham) and the type of stimulus (PInt or CInt) as within-participant factors, and sex as the between-participants factor. Sensation scores were compared between active tDCS and sham tDCS using a Wilcoxon matched-pair test. Statistical significance was set at $p<0.05$.

Finally, a regression analysis was performed for correlations between ToM performance (AI task mean performance in the sham tDCS condition and the RME score) and neuropsychological assessment measures in order to investigate the relationship between ToM and cognitive abilities. We also performed regression analysis between active tDCS-induced changes in the AI task (RTs in the sham tDCS condition subtracted by those in the active $\mathrm{tDCS}$ condition) and neuropsychological assessment measures to determine whether greater difficulties in some cognitive abilities influenced the effects of tDCS on ToM. Statistical significance for regression analysis was set at $p<0.01$ (Bonferroni corrected for the number of comparisons, $p=0.05 / 5=0.01$ ) for clinical scales, at $p<0.013$ (Bonferroni corrected for the number of comparisons, $p=0.05 / 4=0.013$ ) for assessments of memory and language and at $p<0.005$ (Bonferroni corrected for the number of comparisons, $p=0.05 / 11=0.005)$ for attentional and executive functions evaluations.

Statistical power and effect size (Cohen's d) analyses were performed using GPower 3.1 [99]. The datasets used and/or analysed during the current study are available from the corresponding author on reasonable request.

\section{Results}

Behavioural performance on ToM tasks in patients with PD-MCl and $\mathrm{HC}$

RME task

Analysis of the RME scores revealed a significant effect of group $\left(\mathrm{F}_{(1,38)}=8.03, p=0.007, \eta^{2}=0.17,1-\beta=0.99\right)$. This result indicates that the PD-MCI group performed 
A

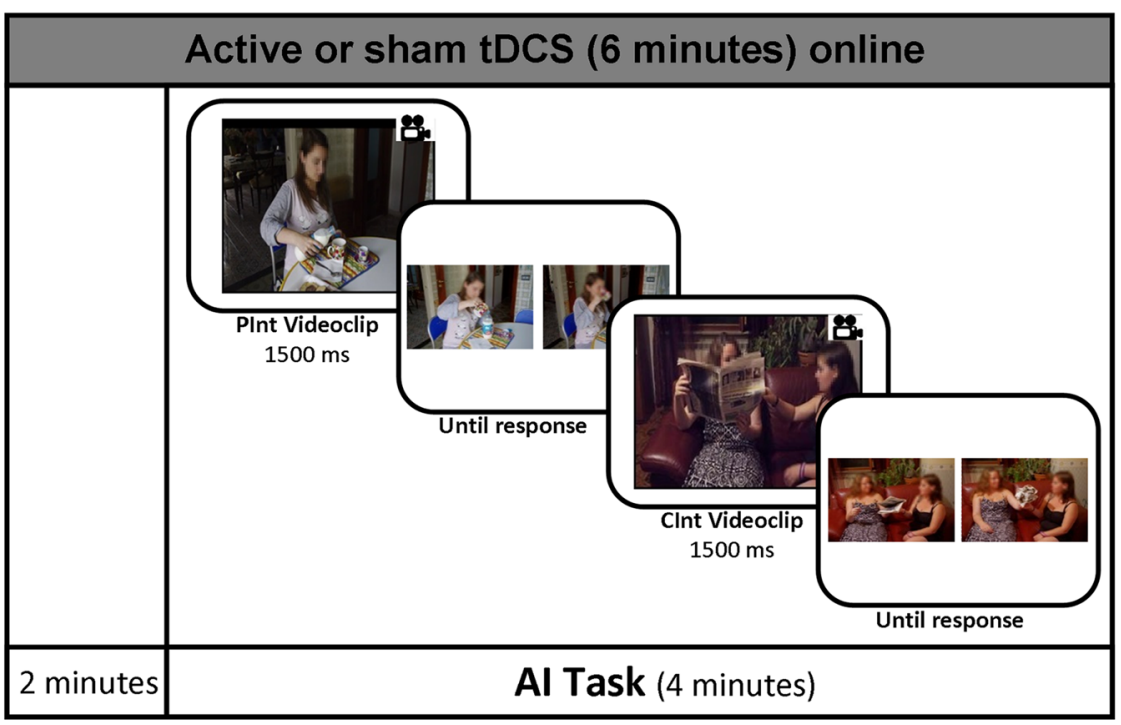

B

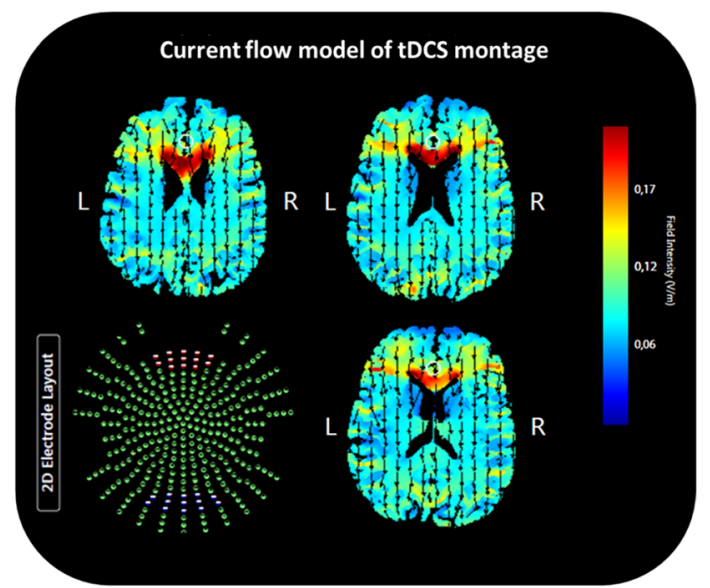

Fig. 1 a Experimental design. Active or sham tDCS was started 2 min before the beginning of the experimental ToM block and continued throughout the Al task. In the Al task, a short video was played, and the participant was asked to choose the picture representing a logical story ending by pushing one of the two buttons on the button box. One example for each stimulus condition (Clnt and PInt) is displayed. $\mathbf{b}$ Current flow model for tDCS. The anode was placed over the medial frontal cortex and the cathode was placed between Inion and Oz. The device utilized two $7-\times 5-\mathrm{cm}$ sponge pads represented in the transverse view on the Male 1 model in Soterix HD Targets software (Soterix Medical). Arrows represent the direction of current flow

worse than the $\mathrm{HC}$ group in this task (HC: $22.9 \pm 3$; PD-MCI: $19.7 \pm 4$ ). See Fig. 2 for details.

\section{Al task}

Accuracy analysis indicated significant effects of group $\left(\mathrm{F}_{(1,38)}=8.53, p=0.006, \eta^{2}=0.18,1-\beta=0.99\right)$ and the type of stimulus $\left(\mathrm{F}_{(1,38)}=4.84, p=0.033, \eta^{2}=0.11,1-\beta=\right.$ $0.99)$. These results indicate that accuracy in the CInt condition was significantly worse than that in the PInt condition in both groups, and that the PD-MCI group had less accuracy in this task than the $\mathrm{HC}$ group did (HC, CInt: $87.1 \% \pm 12$, PInt: $94.4 \% \pm 6$; PD-MCI, CInt: $79.1 \% \pm 16 \%$, PInt: $80.3 \% \pm 16 \%)$.

RT analysis revealed a significant effect of group $\left(\mathrm{F}_{(1,38)}=6.31, p=0.016, \eta^{2}=0.14,1-\beta=0.99\right)$. This result indicates that the PD-MCI group was slower than the $\mathrm{HC}$ group in both CInt and PInt conditions (HC, CInt: $1757.0 \pm 387 \mathrm{~ms}$, PInt: $1742.2 \pm 321 \mathrm{~ms}$; PD-MCI, CInt: $2391.1 \pm 1042 \mathrm{~ms}$, PInt: $2370.5 \pm 1084 \mathrm{~ms})$. No other effect reached statistical significance. See Fig. 3 for details.

Effects of tDCS on the Al task in patients with PD-MCI

Accuracy analysis revealed no significant effects of the type of stimulation $\left(\mathrm{F}_{(1,18)}=1.31, p=0.27, \eta^{2}=0.06\right.$, $1-\beta=0.18)$, the type of stimulus $\left(\mathrm{F}_{(1,18)}=1.37, p=0.26\right.$, $\left.\eta^{2}=0.07,1-\beta=0.65\right)$, or sex $\left(\mathrm{F}_{(1,18)}=3.87, p=0.07, \eta^{2}\right.$ $=0.17,1-\beta=0.65)$, or interactions between factors (CInt condition: $79 \% \pm 16 \%$ [sham tDCS], $75 \% \pm 18 \%$ [active tDCS]; PInt condition: $80 \% \pm 15 \%$ [sham tDCS], $78 \% \pm 18 \%$ [active tDCS]). 

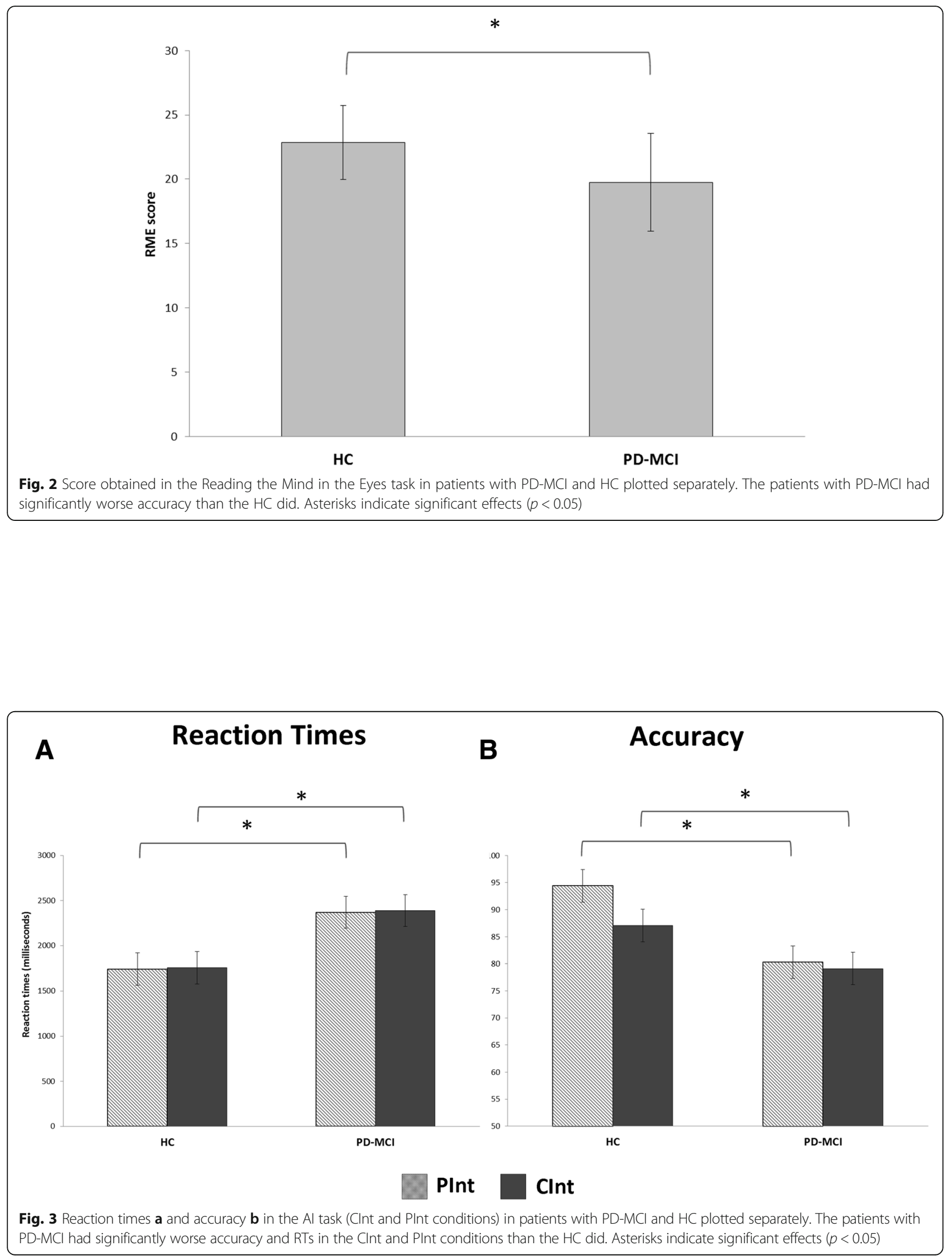
RT analysis revealed a significant effect of the type of stimulation (active vs. sham) $\left(\mathrm{F}_{(1,18)}=6.21, p=0.022\right.$, $\left.\eta^{2}=0.26,1-\beta=0.99\right)$. No other effect reached statistical significance. These results indicate active tDCS induced shorter RTs than sham tDCS in both CInt and PInt conditions (Active tDCS, CInt: $2096.1 \pm 727 \mathrm{~ms}$, PInt: $2096.0 \pm 939 \mathrm{~ms}$; Sham tDCS, CInt: $2391.1 \pm 1042 \mathrm{~ms}$, PInt: $2370.5 \pm 1084 \mathrm{~ms}$ ) (Fig. 4).

\section{Sensations questionnaire}

Responses to the sensations questionnaire completed by patients with PD-MCI at the end of each stimulation session revealed that all of the patients tolerated the stimulation well. A Wilcoxon matched pairs test revealed that perceptual sensations reported after the active and sham stimulation sessions were not significantly different ( $T=13.5, \mathrm{z}=1.73 ; p=0.08)$. There was thus no reason to reject the blinded nature of this study.

\section{Correlation analysis between ToM performance and} apathy, alexithymia, and neuropsychological assessment in patients with PD-MCl

We used ToM performance (AI task in sham tDCS condition and RME score) as the predictor variable and apathy, alexithymia, or neuropsychological assessment as the criterion variable. No significant correlations were found with RME score as the predictor variable.
AI task RT predicted performance on the Stroop task (Time: $r=0.80, p<0.0001$; Errors: $r=0.79, p<0.0001$ ), performance on the FAB $(r=-0.67, p=0.003)$ and time score of the Response Flexibility task of TEA battery $(r=0.70, p=0.002)$, with poorer frontal-executive abilities associated with longer RTs in the AI task (see Fig. 5). No significant correlations were found between AI task accuracy and apathy, alexithymia, or other neuropsychological scores.

\section{Correlation analysis between active tDCS-induced} changes in the Al task and apathy, alexithymia, and neuropsychological assessment in patients with PD-MCI Apathy, alexithymia, or neuropsychological assessment was used as the predictor variable, and active tDCS-induced changes in the AI task were used as the criterion variable.

No significant correlations were found.

\section{Discussion}

The aims of the present study were threefold. We hypothesized that, in patients with PD-MCI, ToM task performance would i) be worse than that in $\mathrm{HC}$, ii) correlate with executive functioning, and iii) be enhanced by active tDCS over the MFC when compared to sham. Our results support the above hypotheses, as follows: i) In the communicative intentions and private

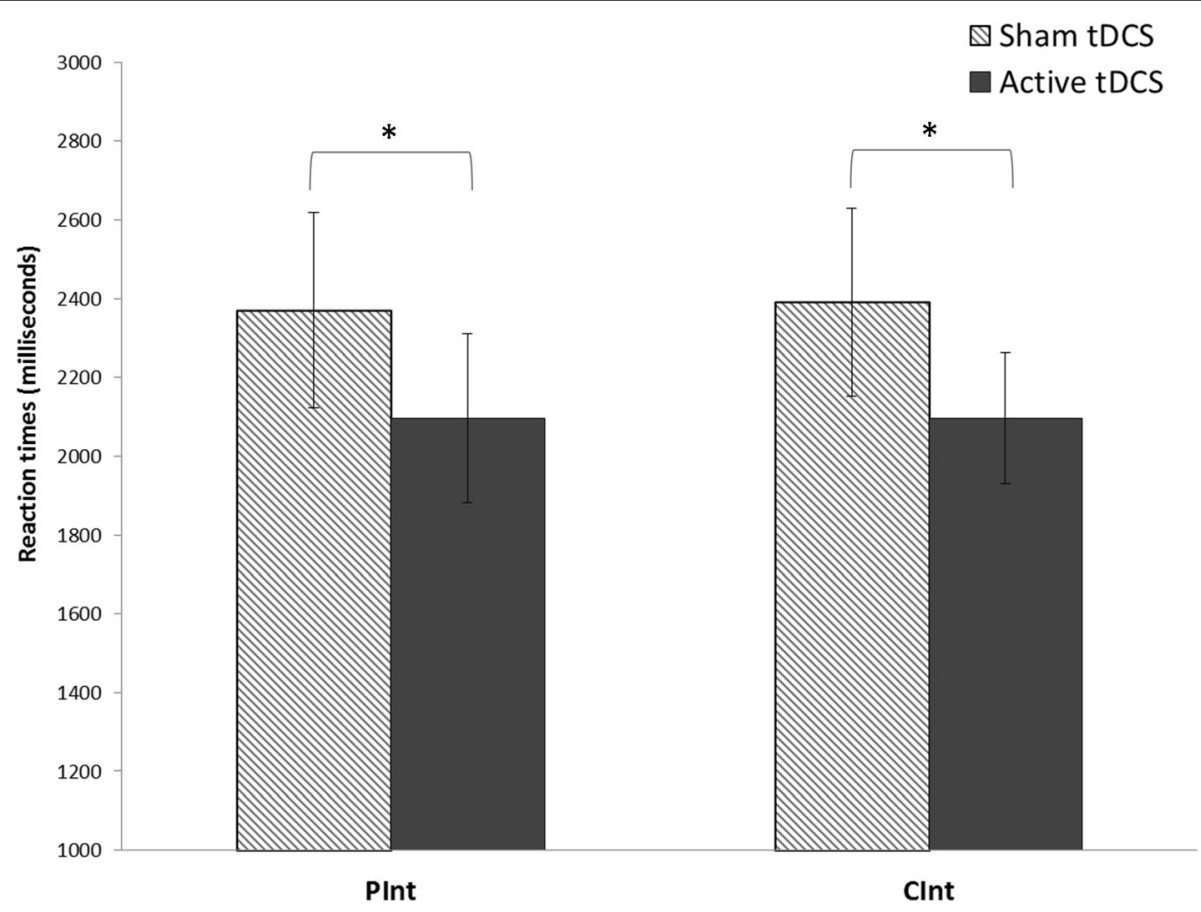

Fig. 4 Effects of tDCS on reaction times in the Al task in patients with PD-MCI under the active tDCS and sham tDCS conditions (Clnt and PInt conditions plotted separately). The reaction times of patients with PD-MCl in the Al task decreased after active tDCS over the MFC (Fpz site, with the cathode between $\mathrm{Oz}$ and Inion) when compared to sham stimulation in both the Clnt and Pint conditions. Asterisks indicate significant effects $(p<0.05)$ 

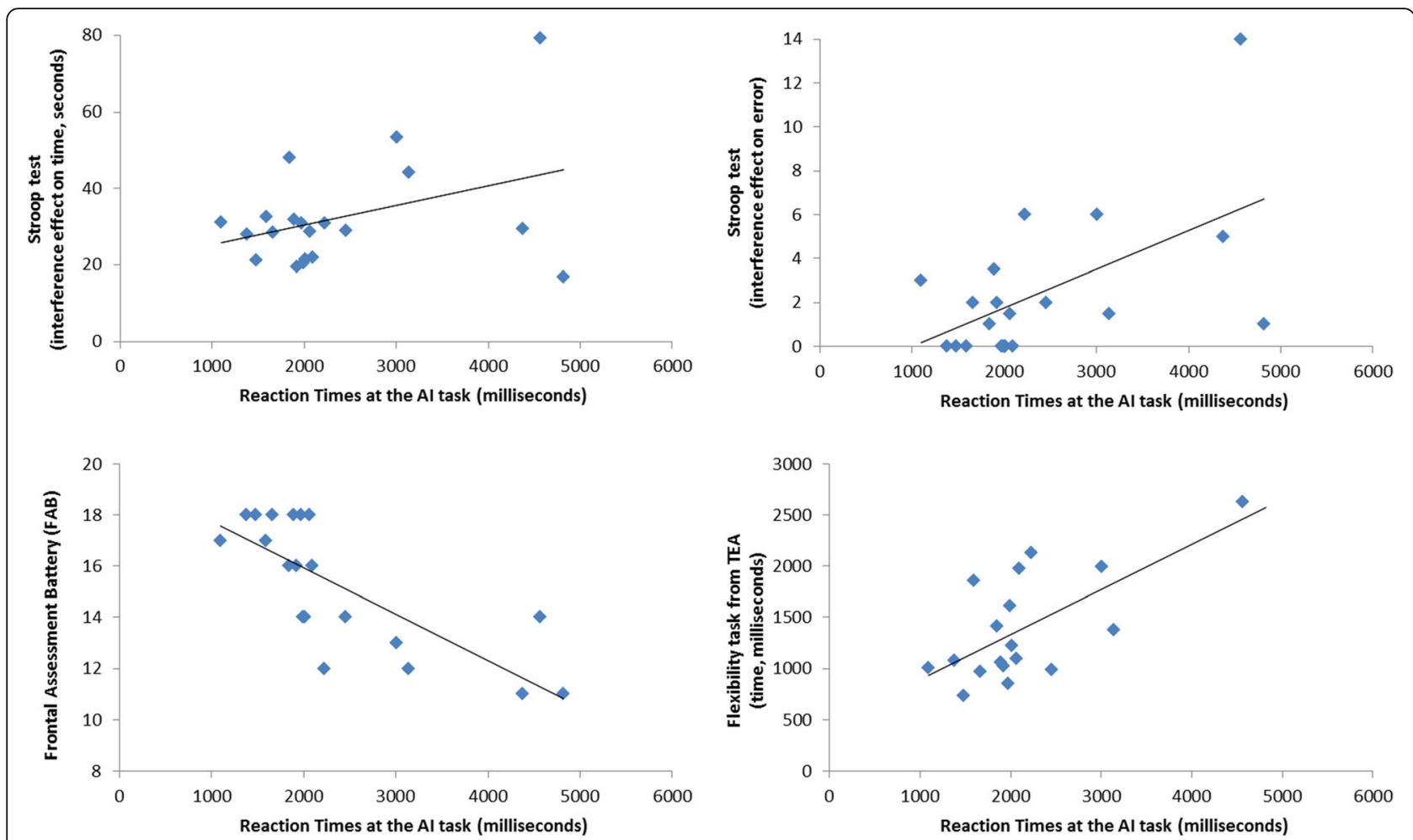

Fig. 5 Significant correlations between reaction time of the Attribution of Intentions task in sham transcranial direct current stimulation condition and attentional-executive abilities in patients with PD-MCI

intentions ToM tasks, the patients were slower and less accurate than the HC. ii) We found relationships between ToM and executive functioning. In particular, ToM abilities were poorer in patients with fronto-executive difficulties as assessed by the FAB and the Stroop task. iii) In patients with PD-MCI, a single session of active tDCS over the MFC led to significant shortening of latency in ToM tasks when compared to sham stimulation. We found no effects of tDCS on accuracy.

Until now, no study had investigated the effects of tDCS on ToM abilities in patients with PD-MCI, and only a few researchers have used tDCS in patients with $\mathrm{PD}$. These researchers mainly focused on the treatment of motor [54, $57,100-104]$ and cognitive symptoms [55, 58, 59, 105].

Our findings corroborate previous studies showing marked difficulties in the domain of ToM in patients with PD [24-29]. In particular, while neuropsychological studies have reported heterogeneous and controversial findings regarding affective ToM ability in patients with PD [106], there is consistent evidence for significant cognitive ToM impairments in these patients [26, 27, 107-109]. As recently shown by Bora, Walterfang, and Velakoulis [110], this may partially be explained by frontal-executive difficulties, which represent a crucial non-motor symptom of patients with PD-MCI. There is a significant relationship between ToM and executive functioning, even though the exact nature of this relationship between these two distinct domains remains at least partially unknown [111, 112]. At any rate, evidence from neuropsychological studies on ToM abilities suggests that executive functioning is necessary to perform cognitive ToM tasks [113-116]. Again, this observation is in line with our finding showing, in a sample of patients with PD-MCI, significant correlations between the RT to the AI task and both the FAB score and the interference effect on time and on error of the Stroop task.

Several lines of evidence suggest that the MFC might be critical for social cognition $[46,117,118]$ and that increased MFC activity might result in enhanced social cognition [46]. The mechanisms underlying the effects of tDCS are not yet entirely understood, but might involve changes in the activities of some neurotransmitters [42, 119]. TDCS modifies the synaptic microenvironment by modifying synaptic strength (i.e., N-methyl-D-aspartate receptor functioning) or altering gamma-aminobutyric acid-mediated activity. It induces transient changes in the densities of protein channels localized to the cortical area below the electrodes, which would in turn interfere with brain excitability through the modulation of intracortical and corticospinal neuron activity [41, 42, 119]. In particular, the activities neurotransmitters, especially that of dopamine, seem to influence the plasticity changes induced by tDCS, as they depend on the relationship between current and the functioning of neurotransmitter receptors [119-121]. 
The finding that a single session of active tDCS over the MFC had a significant effect on ToM performance in patients with PD-MCI might have important clinical implications. In fact, evidence for enhancement of ToM performance by tDCS might lead to a potential intervention approach for this disease. However, we acknowledge that the present study has some limitations. First, it is important to underline that the enhancement we found was limited to RTs and that we observed no effect on accuracy. Another shortcoming is that we classified PD-MCI through a global scale (PD-CRS; [3]). A limitation in terms of the stimulation protocol is that we applied a single-session online tDCS design that focuses on short-term improvements induced by a single session of stimulation, typically delivered on-line during the task. The adoption of multiple sessions of tDCS could be used to investigate the long-term effects of stimulation, which are particularly interesting in neurodegenerative patients. Hence, further studies are needed in order to conclusively demonstrate the potential for the induction of long-term neuromodulatory effects using brain stimulation. In particular, the same protocol might be applied to PD-MCI patients to elucidate long-term improvements in ToM performance. Moreover, future studies including larger cohorts of patients at different stages of disease should be conducted before firm conclusion can be drawn. These interesting studies could better investigate the possibility to induce also effects on accuracy in addition to reaction times modulation. As known, tDCS is a non-invasive brain stimulation technique characterized by limited focality and several methodological factors, such as type of protocols design, target area, polarity and number of sessions, that can potentially influence the variability of behavioural and physiological outcomes $[42,122]$. Accordingly, a further limit is represented by the lack of a control active stimulation site that should be considered in future studies to further confirm the specificity of the MFC for the present results on ToM tasks.

\section{Conclusion}

Despite the limitations described, our findings show for the first time that active tDCS applied over the MFC could be useful for enhancing cognitive ToM ability in patients with PD-MCI. Our findings suggest that non-invasive brain stimulation could be used in an attempt to ameliorate the ToM deficits observed in these patients. Of course, further studies are needed to determine whether the effects found here are clinically meaningful. Nevertheless, the present study significantly contributes to the emergent evidence suggesting that tDCS may be used to treat PD $[44,123]$ and to improve social cognition [51, 124].

\section{Abbreviations}

BDI-II: Beck Depression Inventory-II; FAB: Frontal assessment battery; HC: Healthy controls; MFC: Medial frontal cortex; MMP: Mini-Mental Parkinson; PD: Parkinson's disease; PD-CRS: Parkinson's disease cognitive rating scale; PD-MCl: Parkinson's disease-mild cognitive impairment; RBDSQ: Rapid eye movement sleep behavior disorders screening questionnaire; TAS-20: Toronto Alexithymia Scale; tDCS: transcranial Direct Current Stimulation; TEA: Test of attentional performance; ToM: Theory of mind; UPDRS: Unified Parkinson's Disease Rating Scale

\section{Acknowledgements}

We wish to thank all the patients and their caregivers for their cooperation.

\section{Funding}

Mauro Adenzato was supported by the University of Turin (Ricerca scientifica finanziata dall'Università "Cognizione sociale e attaccamento in popolazioni cliniche e non cliniche"). Ivan Enrici was supported by University of Turin grants (Ricerca scientifica finanziata dall'Università "Linea Generale" and "Linea Giovani").

\section{Availability of data and materials}

The dataset used and/or analysed during the current study are available from the corresponding author on reasonable request.

\section{Authors' contributions}

Study concept and design: MA, RM, IE, BB, MC. Data acquisition: RM, EG, MB, AA, MSC, AP, BB, MC. Data analysis and interpretation: MA, RM, IE, MC. Drafting of the manuscript: MA, RM, IE, BB, MC. Statistical Analysis: RM, MC. Study supervision: MA, MC. All authors read and approved the final manuscript.

Ethics approval and consent to participate

All procedures performed in studies involving human participants were in accordance with the ethical standards of the institutional research committee. Informed consent was obtained from all individual participants included in the study. Ethics approval was obtained from the local Ethical Committee (IRCCS Istituto Centro San Giovanni di Dio Fatebenefratelli, Brescia, Italy).

\section{Consent for publication}

Not applicable

\section{Competing interests}

The authors declare that they have no competing interests.

\section{Author details}

${ }^{1}$ Department of Psychology, University of Turin, Turin, Italy. ${ }^{2}$ Neuroscience Institute of Turin, Turin, Italy. ${ }^{3}$ Neuropsychology Unit, IRCCS Istituto Centro San Giovanni di Dio - Fatebenefratelli, Brescia, Italy. ${ }^{4}$ Department of Philosophy and Educational Sciences, University of Turin, via Gaudenzio Ferrari 9, 10124 Turin, Italy. ${ }^{5}$ Centre for Neurodegenerative Disorders, Neurology Unit, Department of Clinical and Experimental Sciences, University of Brescia, Brescia, Italy.

Received: 31 July 2018 Accepted: 18 December 2018 Published online: 07 January 2019

\section{References}

1. Mayeux R. Epidemiology of neurodegeneration. Annu Rev Neurosci. 2003; 26:81-104.

2. Chaudhuri KR, Schapira AH. Non-motor symptoms of Parkinson's disease: dopaminergic pathophysiology and treatment. Lancet Neurol. 2009:8:464-74.

3. Litvan I, Goldman JG, Troster Al, Schmand BA, Weintraub D, Petersen RC, et al. Diagnostic criteria for mild cognitive impairment in Parkinson's disease: Movement Disorder Society task force guidelines. Mov Disord. 2012;27:349-56.

4. Aarsland D, Creese B, Politis M, Chaudhuri KR, Ffytche DH, Weintraub D, et al. Cognitive decline in Parkinson disease. Nat Rev Neurol. 2017;13: 217-31

5. Kalia LV, Lang AE. Parkinson's disease. Lancet. 2015;386:896-912.

6. Lees AJ, Hardy J, Revesz T. Parkinson's disease. Lancet. 2009;373:2055-66.

7. Aarsland D, Kramberger MG. Neuropsychiatric symptoms in Parkinson's disease. J Parkinsons Dis. 2015;5:659-67. 
8. Dubois B, Pillon B. Cognitive deficits in Parkinson's disease. J Neurol. 1997;244:2-8.

9. Goldman JG, Litvan I. Mild cognitive impairment in Parkinson's disease Minerva Med. 2011;102:441-59.

10. Kehagia AA, Barker RA, Robbins TW. Neuropsychological and clinical heterogeneity of cognitive impairment and dementia in patients with Parkinson's disease. Lancet Neurol. 2010;9:1200-13.

11. Padovani A, Costanzi C, Gilberti N, Borroni B. Parkinson's disease and dementia. Neurol Sci. 2006;27(Suppl 1):S40-3.

12. Pagonabarraga J, Kulisevsky J. Cognitive impairment and dementia in Parkinson's disease. Neurobiol Dis. 2012:46:590-6.

13. Gallagher DA, Lees AJ, Schrag A. What are the most important nonmotor symptoms in patients with Parkinson's disease and are we missing them? Mov Disord. 2010;25:2493-500

14. Geurtsen GJ, Hoogland J, Goldman JG, Schmand BA, Troster Al, Burn DJ, et al. Parkinson's disease mild cognitive impairment: application and validation of the criteria. J Parkinsons Dis. 2014;4:131-7.

15. Emre M, Aarsland D, Brown R, Burn DJ, Duyckaerts C, Mizuno Y, et al. Clinical diagnostic criteria for dementia associated with Parkinson's disease. Mov Disord. 2007;22:1689-707 quiz 837.

16. Enrici I, Adenzato M, Ardito RB, Mitkova A, Cavallo M, Zibetti M, et al. Emotion processing in Parkinson's disease: a three-level study on recognition, representation, and regulation. PLoS One. 2015;10:e0131470.

17. Biundo R, Fiorenzato E, Antonini A. Nonmotor symptoms and natural history of Parkinson's disease: evidence from cognitive dysfunction and role of noninvasive interventions. Int Rev Neurobiol. 2017:133:389-415.

18. Litvan I, Aarsland D, Adler CH, Goldman JG, Kulisevsky J, Mollenhauer B, et al. MDS task force on mild cognitive impairment in Parkinson's disease: critical review of PD-MCI. Mov Disord. 2011;26:1814-24.

19. Weintraub D, Troster Al, Marras C, Stebbins G. Initial cognitive changes in Parkinson's disease. Mov Disord 2018;33(4):511-519.

20. Yarnall AJ, Breen DP, Duncan GW, Khoo TK, Coleman SY, Firbank MJ, et al. Characterizing mild cognitive impairment in incident Parkinson disease: the ICICLE-PD study. Neurology. 2014;82:308-16.

21. Marras C, Armstrong MJ, Meaney CA, Fox S, Rothberg B, Reginold W, et al. Measuring mild cognitive impairment in patients with Parkinson's disease. Mov Disord. 2013:28:626-33.

22. Goldman JG, Holden S, Bernard B, Ouyang B, Goetz CG, Stebbins GT. Defining optimal cutoff scores for cognitive impairment using Movement Disorder Society task force criteria for mild cognitive impairment in Parkinson's disease. Mov Disord. 2013:28:1972-9.

23. Lawrence BJ, Gasson N, Loftus AM. Prevalence and subtypes of mild cognitive impairment in Parkinson's disease. Sci Rep. 2016;6:33929.

24. Poletti M, Enrici I, Adenzato M. Cognitive and affective theory of mind in neurodegenerative diseases: neuropsychological, neuroanatomical and neurochemical levels. Neurosci Biobehav Rev. 2012;36:2147-64.

25. Bodden ME, Dodel R, Kalbe E. Theory of mind in Parkinson's disease and related basal ganglia disorders: a systematic review. Mov Disord. 2010;25:13-27.

26. Bodden ME, Mollenhauer B, Trenkwalder C, Cabanel N, Eggert KM, Unger $\mathrm{MM}$, et al. Affective and cognitive theory of mind in patients with Parkinson's disease. Parkinsonism Relat Disord. 2010;16:466-70.

27. Roca M, Torralva T, Gleichgerrcht E, Chade A, Arévalo GG, Gershanik O, et al. Impairments in Social Cognition in Early Medicated and Unmedicated Parkinson Disease. Cog Behav Neurol. 2010;23:152-8.

28. Santangelo G, Vitale C, Trojano L, Errico D, Amboni M, Barbarulo AM, et al. Neuropsychological correlates of theory of mind in patients with early Parkinson's disease. Mov Disord. 2012;27:98-105.

29. Tsuruya N, Kobayakawa M, Kawamura M. Is "reading mind in the eyes" impaired in Parkinson's disease? Parkinsonism Relat Disord. 2011;17:246-8.

30. Carrington SJ, Bailey AJ. Are there theory of mind regions in the brain? A review of the neuroimaging literature. Hum Brain Mapp. 2009;30:2313-35.

31. Molenberghs $P$, Johnson H, Henry JD, Mattingley JB. Understanding the minds of others: a neuroimaging meta-analysis. Neurosci Biobehav Rev. 2016;65:276-91

32. Van Overwalle F. Social cognition and the brain: a meta-analysis. Hum Brain Mapp. 2009;30:829-58

33. Adenzato M, Cavallo M, Enrici I. Theory of mind ability in the behavioural variant of frontotemporal dementia: an analysis of the neural, cognitive, and social levels. Neuropsychologia. 2010;48:2-12.

34. Adenzato $\mathrm{M}$, Poletti $\mathrm{M}$. Theory of mind abilities in neurodegenerative diseases: an update and a call to introduce mentalizing tasks in standard neuropsychological assessments. Clin Neuropsychiatry. 2013;10.
35. Brioschi Guevara A, Knutson KM, Wassermann EM, Pulaski S, Grafman J, Krueger $\mathrm{F}$. Theory of mind impairment in patients with behavioural variant fronto-temporal dementia (bv-FTD) increases caregiver burden. Age Ageing. 2015:44:891-5.

36. Gregory C, Lough S, Stone V, Erzinclioglu S, Martin L, Baron-Cohen S, et al. Theory of mind in patients with frontal variant frontotemporal dementia and Alzheimer's disease: theoretical and practical implications. Brain. 2002 125:752-64.

37. Henry JD, Phillips LH, von Hippel C. A meta-analytic review of theory of mind difficulties in behavioural-variant frontotemporal dementia. Neuropsychologia. 2014;56:53-62.

38. Poletti M, Enrici I, Bonuccelli U, Adenzato M. Theory of mind in Parkinson's disease. Behav Brain Res. 2011:219:342-50.

39. Yu RL, Wu RM. Social brain dysfunctions in patients with Parkinson's disease: a review of theory of mind studies. Transl Neurodegener. 2013;2:7.

40. Nitsche MA, Cohen LG, Wassermann EM, Priori A, Lang N, Antal A, et al. Transcranial direct current stimulation: state of the art 2008. Brain Stimul. 2008;1:206-23.

41. Paulus W. Transcranial electrical stimulation (tES - tDCS; tACS; tRNS) methods. Neuropsychological Rehabilitation. 2011;21:602-17.

42. Polania R, Nitsche MA, Ruff CC. Studying and modifying brain function with non-invasive brain stimulation. Nat Neurosci. 2018;21:174-87.

43. Vallar G, Bolognini N. Behavioural facilitation following brain stimulation: implications for neurorehabilitation. Neuropsychol Rehabil. 2011;21:618-49.

44. Brunoni AR, Nitsche MA, Bolognini N, Bikson M, Wagner T, Merabet L, et al. Clinical research with transcranial direct current stimulation (tDCS): challenges and future directions. Brain Stimul. 2012;5:175-95.

45. Martins AR, Fregni F, Simis M, Almeida J. Neuromodulation as a cognitive enhancement strategy in healthy older adults: promises and pitfalls. Neuropsychol Dev Cogn B Aging Neuropsychol Cogn. 2017;24:158-85.

46. Adenzato M, Brambilla M, Manenti R, De Lucia L, Trojano L, Garofalo S, et al. Gender differences in cognitive theory of mind revealed by transcranial direct current stimulation on medial prefrontal cortex. Sci Rep. 2017;7:41219.

47. Conson M, Errico D, Mazzarella E, Giordano M, Grossi D, Trojano L. Transcranial electrical stimulation over dorsolateral prefrontal cortex modulates processing of social cognitive and affective information. PLoS One. 2015:10:e0126448.

48. Fumagalli M, Vergari M, Pasqualetti P, Marceglia S, Mameli F, Ferrucci R, et al. Brain switches utilitarian behavior: does gender make the difference? PLoS One. 2010;5:e8865.

49. Mai X, Zhang W, Hu X, Zhen Z, Xu Z, Zhang J, et al. Using tDCS to explore the role of the right Temporo-parietal junction in theory of mind and cognitive empathy. Front Psychol. 2016;7:380.

50. Martin AK, Huang J, Hunold A, Meinzer M. Sex mediates the effects of highdefinition transcranial direct current stimulation on "mind-Reading". Neuroscience. 2017:366:84-94.

51. Santiesteban I, Banissy MJ, Catmur C, Bird G. Enhancing social ability by stimulating right temporoparietal junction. Curr Biol. 2012;22:2274-7.

52. Martin AK, Dzafic I, Ramdave S, Meinzer M. Causal evidence for task-specific involvement of the dorsomedial prefrontal cortex in human social cognition. Soc Cogn Affect Neurosci. 2017;12:1209-18.

53. Liao C, Wu S, Luo YJ, Guan Q Cui F. Transcranial direct current stimulation of the medial prefrontal cortex modulates the propensity to help in costly helping behavior. Neurosci Lett. 2018:674:54-9.

54. Manenti R, Brambilla M, Benussi A, Rosini S, Cobelli C, Ferrari C, et al. Mild cognitive impairment in Parkinson's disease is improved by transcranial direct current stimulation combined with physical therapy. Mov Disord. 2016;31:715-24.

55. Doruk D, Gray Z, Bravo GL, Pascual-Leone A, Fregni F. Effects of tDCS on executive function in Parkinson's disease. Neurosci Lett. 2014:582:27-31.

56. Dinkelbach L, Brambilla M, Manenti R, Brem AK. Non-invasive brain stimulation in Parkinson's disease: exploiting crossroads of cognition and mood. Neurosci Biobehav Rev. 2017:75:407-18.

57. Benninger DH, Lomarev M, Lopez G, Wassermann EM, Li X, Considine E, et al. Transcranial direct current stimulation for the treatment of Parkinson's disease. J Neurol Neurosurg Psychiatry. 2010;81:1105-11.

58. Biundo R, Weis L, Fiorenzato E, Gentile G, Giglio M, Schifano R, et al. Double-blind randomized trial of tDCS versus sham in Parkinson patients with mild cognitive impairment receiving cognitive training. Brain Stimul. 2015;8:1223-5. 
59. Boggio PS, Ferrucci R, Rigonatti SP, Covre P, Nitsche M, Pascual-Leone A, et al. Effects of transcranial direct current stimulation on working memory in patients with Parkinson's disease. J Neurol Sci. 2006;249:31-8.

60. Mally J, Stone TW, Sinko G, Geisz N, Dinya E. Long term follow-up study of non-invasive brain stimulation (NBS) (rTMS and tDCS) in Parkinson's disease (PD). Strong age-dependency in the effect of NBS. Brain Res Bull. 2018;142: 78-87.

61. Bara $\mathrm{BG}$, Ciaramidaro A, Walter $\mathrm{H}$, Adenzato M. Intentional minds: a philosophical analysis of intention tested through fMRI experiments involving people with schizophrenia, people with autism, and healthy individuals. Front Hum Neurosci. 2011:5:7.

62. Ciaramidaro A, Adenzato M, Enrici I, Erk S, Pia L, Bara BG, et al. The intentional network: how the brain reads varieties of intentions. Neuropsychologia. 2007:45:3105-13

63. Walter H, Adenzato M, Ciaramidaro A, Enrici I, Pia L, Bara BG. Understanding intentions in social interaction: the role of the anterior paracingulate cortex. J Cogn Neurosci. 2004;16:1854-63.

64. Walter H, Ciaramidaro A, Adenzato M, Vasic N, Ardito RB, Erk S, et al. Dysfunction of the social brain in schizophrenia is modulated by intention type: an fMRI study. Soc Cogn Affect Neurosci. 2009;4:166-76.

65. Postuma RB, Berg D, Stern M, Poewe W, Olanow CW, Oertel W, et al. MDS clinical diagnostic criteria for Parkinson's disease. Mov Disord. 2015;30:1591-601.

66. Hughes AJ, Daniel SE, Kilford L, Lees AJ. Accuracy of clinical diagnosis of idiopathic Parkinson's disease: a clinico-pathological study of 100 cases. J Neurol Neurosurg Psychiatry. 1992;55:181-4.

67. Santangelo G, Barone P, Abbruzzese G, Ferini-Strambi L, Antonini A, Group IS. Validation of the Italian version of Parkinson's disease-cognitive rating scale (PD-CRS). Neurol Sci. 2014;35:537-44.

68. Costa A, Bagoj E, Monaco M, Zabberoni S, De Rosa S, Mundi C, et al. Mini mental Parkinson test: standardization and normative data on an Italian sample. Neurol Sci. 2013;34:1797-803

69. Skorvanek M, Goldman JG, Jahanshahi M, Marras C, Rektorova I, Schmand B, et al. Global scales for cognitive screening in Parkinson's disease: critique and recommendations. Mov Disord. 2018;33:208-18.

70. Poreisz C, Boros K, Antal A, Paulus W. Safety aspects of transcranial direct current stimulation concerning healthy subjects and patients. Brain Res Bull. 2007;72:208-14

71. Pagonabarraga J, Kulisevsky J, Llebaria G, Garcia-Sanchez C, Pascual-Sedano B, Gironell A. Parkinson's disease-cognitive rating scale: a new cognitive scale specific for Parkinson's disease. Mov Disord. 2008;23:998-1005.

72. Sandrini M, Manenti R, Brambilla M, Cobelli C, Cohen LG, Cotelli M. Older adults get episodic memory boosting from noninvasive stimulation of prefrontal cortex during learning. Neurobiol Aging. 2016;39:210-6.

73. Fernandez de Bobadilla R, Pagonabarraga J, Martinez-Horta S, PascualSedano B, Campolongo A, Kulisevsky J. Parkinson's disease-cognitive rating scale: psychometrics for mild cognitive impairment. Mov Disord. 2013;28: 1376-83.

74. Di Virgilio G, Leroy A, Cunin P, Mahieux F, Bachoud-Levi A, Fenelon G. The mini mental Parkinson brief cognitive test: comparison with the Mattis dementia rating scale in 289 patients with Parkinson's disease. Mov Disord. 2007;22:590

75. Mahieux F, Michelet D, Manifacier MJ, Boller F, Fermanian J, Guillard A. Minimental Parkinson: first validation study of a new bedside test constructed for Parkinson's disease. Behav Neurol. 1995;8:15-22.

76. Aarsland D, Cummings J, Weintraub D, Chaudhuri KR. Neuropsychiatric and cognitive changes in Parkinson's disease and related movement disorders: diagnosis and management: Cambridge University Press; 2013.

77. Kulisevsky J, Fernandez de Bobadilla R, Pagonabarraga J, Martinez-Horta S, Campolongo A, Garcia-Sanchez C, et al. Measuring functional impact of cognitive impairment: validation of the Parkinson's disease cognitive functional rating scale. Parkinsonism Relat Disord. 2013;19:812-7.

78. Kulisevsky J, Pagonabarraga J. Cognitive impairment in Parkinson's disease: tools for diagnosis and assessment. Mov Disord. 2009;24:1103-10.

79. Bates E, Andonova E, D'Amico S, Jacobsen T, Kohnert K, Lu CC, et al. Introducing the CRL international picture-naming project (CRL-IPNP). University of California San Diego: Center for Research in Language Newsletter La Jolla; 2000. p. 12.

80. Zimmermann P, Fimm B. A test battery for attentional performance. Applied neuropsychology of attention Theory, diagnosis and rehabilitation; 2002. p. $110-51$.
81. Appollonio I, Leone M, Isella V, Piamarta F, Consoli T, Villa ML, et al. The frontal assessment battery (FAB): normative values in an Italian population sample. Neurol Sci. 2005;26:108-16.

82. Lezak M, Howieson D, Loring DW. Neuropsychological assessment (fourth edition). Oxford: University Press; 2004.

83. Beck AT, Steer RA, Ball R, Ranieri W. Comparison of Beck depression inventories -IA and -II in psychiatric outpatients. J Pers Assess. 1996;67:588-97.

84. Jenkinson C, Fitzpatrick R, Peto V, Greenhall R, Hyman N. The Parkinson's disease questionnaire (PDQ-39): development and validation of a Parkinson's disease summary index score. Age Ageing. 1997;26:353-7.

85. Steinberg L, Sharp C, Stanford MS, Tharp AT. New tricks for an old measure: the development of the Barratt impulsiveness scale-brief (BIS-brief). Psychol Assess. 2013:25:216-26.

86. Santangelo G, Barone P, Cuoco S, Raimo S, Pezzella D, Picillo M, et al. Apathy in untreated, de novo patients with Parkinson's disease: validation study of apathy evaluation scale. J Neurol. 2014;261:2319-28.

87. Stiasny-Kolster K, Mayer G, Schafer S, Moller JC, Heinzel-Gutenbrunner M, Oertel WH. The REM sleep behavior disorder screening questionnaire: a new diagnostic instrument. Mov Disord. 2007;22:2386-93.

88. Antonini A, Abbruzzese G, Ferini-Strambi L, Tilley B, Huang J, Stebbins GT, et al. Validation of the Italian version of the Movement Disorder Society-unified Parkinson's disease rating scale. Neurol Sci. 2013;34:683-7.

89. Bressi C, Taylor G, Parker J, Bressi S, Brambilla V, Aguglia E, et al. Cross validation of the factor structure of the 20-item Toronto alexithymia scale: an Italian multicenter study. J Psychosom Res. 1996:41:551-9.

90. Albiero P, Ingoglia S, Lo CA. Contributo all'adattamento italiano dell'Interpersonal Reactivity Index. Testing Psicometria Metodologia. 2006;13.

91. Hoehn MM, Yahr MD. Parkinsonism: onset, progression and mortality. Neurology. 1967;17:427-42.

92. Nitsche MA, Doemkes S, Karakose T, Antal A, Liebetanz D, Lang N, et al. Shaping the effects of transcranial direct current stimulation of the human motor cortex. J Neurophysiol. 2007:97:3109-17.

93. Nitsche MA, Liebetanz D, Lang N, Antal A, Tergau F, Paulus W. Safety criteria for transcranial direct current stimulation (tDCS) in humans. Clin Neurophysiol. 2003;114:2220-2 author reply 2-3.

94. Nitsche MA, Paulus W. Excitability changes induced in the human moto cortex by weak transcranial direct current stimulation. J Physiol. 2000;527(Pt 3):633-9.

95. Baron-Cohen S, Wheelwright S, Hill J, Raste Y, Plumb I. The "Reading the mind in the eyes" test revised version: a study with normal adults, and adults with Asperger syndrome or high-functioning autism. J Child Psychol Psychiatry. 2001;42:241-51.

96. Enrici I, Adenzato M, Cappa S, Bara BG, Tettamanti M. Intention processing in communication: a common brain network for language and gestures. J Cogn Neurosci. 2011;23:2415-31.

97. Tettamanti M, Vaghi MM, Bara BG, Cappa SF, Enrici I, Adenzato M. Effective connectivity gateways to the theory of mind network in processing communicative intention. Neuroimage. 2017;155:169-76.

98. Herwig U, Satrapi P, Schonfeldt-Lecuona C. Using the international 10-20 EEG system for positioning of transcranial magnetic stimulation. Brain Topogr. 2003;16:95-9.

99. Faul F, Erdfelder E, Lang AG, Buchner A. G*power 3: a flexible statistical power analysis program for the social, behavioral, and biomedical sciences. Behav Res Methods. 2007;39:175-91.

100. del Olmo MF, Bello O, Cudeiro J. Transcranial magnetic stimulation over dorsolateral prefrontal cortex in Parkinson's disease. Clin Neurophysiol. 2007; 118:131-9.

101. Fregni F, Boggio PS, Santos MC, Lima M, Vieira AL, Rigonatti SP, et al. Noninvasive cortical stimulation with transcranial direct current stimulation in Parkinson's disease. Mov Disord. 2006:21:1693-702.

102. Kaski D, Dominguez RO, Allum JH, Islam AF, Bronstein AM. Combining physical training with transcranial direct current stimulation to improve gait in Parkinson's disease: a pilot randomized controlled study. Clin Rehabil. 2014:28:1115-24.

103. Manenti R, Brambilla M, Rosini S, Orizio I, Ferrari C, Borroni B, et al. Time up and go task performance improves after transcranial direct current stimulation in patient affected by Parkinson's disease. Neurosci Lett. 2014; 580:74-7.

104. Verheyden G, Purdey J, Burnett M, Cole J, Ashburn A. Immediate effect of transcranial direct current stimulation on postural stability and functional mobility in Parkinson's disease. Mov Disord. 2013;28:2040-1. 
105. Pereira JB, Junque C, Bartres-Faz D, Marti MJ, Sala-Llonch R, Compta Y, et al. Modulation of verbal fluency networks by transcranial direct current stimulation (tDCS) in Parkinson's disease. Brain Stimul. 2013;6:16-24.

106. Poletti M, Bonuccelli U. Orbital and ventromedial prefrontal cortex functioning in Parkinson's disease: neuropsychological evidence. Brain Cogn. 2012;79:23-33.

107. Kawamura M. Social cognitive impairment is an integral part of Parkinson disease. Rinsho Shinkeigaku. 2011;51:1-5.

108. Monetta L, Grindrod CM, Pell MD. Irony comprehension and theory of mind deficits in patients with Parkinson's disease. Cortex. 2009;45:972-81.

109. Peron J, Vicente S, Leray E, Drapier S, Drapier D, Cohen R, et al. Are dopaminergic pathways involved in theory of mind? A study in Parkinson's disease. Neuropsychologia. 2009;47:406-14.

110. Bora E, Walterfang M, Velakoulis D. Theory of mind in Parkinson's disease: a meta-analysis. Behav Brain Res. 2015:292:515-20.

111. Bird CM, Castelli F, Malik O, Frith U, Husain M. The impact of extensive medial frontal lobe damage on 'Theory of Mind' and cognition. Brain. 2004; 127:914-28.

112. Di Tella M, Castelli L, Colonna F, Fusaro E, Torta R, Ardito RB, et al. Theory of mind and emotional functioning in fibromyalgia syndrome: an investigation of the relationship between social cognition and executive function. PLoS One. 2015:10:e0116542.

113. Carlson SM, Moses L, Breton C. How specific is the relation between executive function and theory of mind? Contributions of inhibitory control and working memory. Infant Child Dev. 2002;11:73-92.

114. Champagne-Lavau M, Joanette Y. Pragmatics, theory of mind and executive functions after a right-hemisphere lesion: different patterns of deficits. J Neurolinguistics. 2009;22:413-26.

115. Dennis M, Agostino A, Roncadin C, Levin H. Theory of mind depends on domain-general executive functions of working memory and cognitive inhibition in children with traumatic brain injury. J Clin Exp Neuropsychol. 2009:31:835-47.

116. Hughes C, Ensor R. Executive function and theory of mind: predictive relations from ages 2 to 4. Dev Psychol. 2007:43:1447.

117. Krause L, Enticott PG, Zangen A, Fitzgerald PB. The role of medial prefrontal cortex in theory of mind: a deep rTMS study. Behav Brain Res. 2012;228:87-90.

118. Lev-Ran S, Shamay-Tsoory SG, Zangen A, Levkovitz Y. Transcranial magnetic stimulation of the ventromedial prefrontal cortex impairs theory of mind learning. Eur Psychiatry. 2012;27:285-9.

119. Dayan E, Censor N, Buch ER, Sandrini M, Cohen LG. Noninvasive brain stimulation: from physiology to network dynamics and back. Nat Neurosci. 2013;16:838-44

120. Monte-Silva K, Liebetanz D, Grundey J, Paulus W, Nitsche MA. Dosagedependent non-linear effect of L-dopa on human motor cortex plasticity. J Physiol. 2010;588:3415-24.

121. Nitsche MA, Muller-Dahlhaus F, Paulus W, Ziemann U. The pharmacology of neuroplasticity induced by non-invasive brain stimulation: building models for the clinical use of CNS active drugs. J Physiol. 2012;590:4641-62.

122. Gandiga PC, Hummel FC, Cohen LG. Transcranial DC stimulation (tDCS): a tool for double-blind sham-controlled clinical studies in brain stimulation. Clin Neurophysiol. 2006;117:845-50.

123. Demirtas-Tatlidede A, Vahabzadeh-Hagh AM, Pascual-Leone A. Can noninvasive brain stimulation enhance cognition in neuropsychiatric disorders? Neuropharmacology. 2013;64:566-78.

124. Sellaro R, Nitsche MA, Colzato LS. The stimulated social brain: effects of transcranial direct current stimulation on social cognition. Ann N Y Acad Sci. 2016;1369:218-39.

\section{Ready to submit your research? Choose BMC and benefit from:}

- fast, convenient online submission

- thorough peer review by experienced researchers in your field

- rapid publication on acceptance

- support for research data, including large and complex data types

- gold Open Access which fosters wider collaboration and increased citations

- maximum visibility for your research: over $100 \mathrm{M}$ website views per year

At $\mathrm{BMC}$, research is always in progress.

Learn more biomedcentral.com/submissions 